\title{
ON THE EXISTENCE OF PERIODIC SOLUTIONS FOR A CLASS OF NONLINEAR DIFFERENTIAL SYSTEMS*
}

\author{
LIHONG HUANG AND JIANSHE YU
}

Abstract. In this paper three theorems on the existence of nontrivial periodic solutions of the system

$$
\begin{aligned}
& d x / d t=e(y) \\
& d y / d t=-e(y) f(x)-g(x)
\end{aligned}
$$

are proved, which not only generalize some known results on the existence of periodic solutions of Liénard's system (i.e. the special form for $e(y)=$ $y$ ), but also relax or eliminate some traditional assumptions.

\section{Introduction}

Consider the nonlinear autonomous differential system

$$
\begin{aligned}
& d x / d t=e(y) \\
& d y / d t=-e(y) f(x)-g(x)
\end{aligned}
$$

where $e(y), f(x)$ and $g(x)$ are continuous real functions defined on $\mathbb{R}$, and are subject to the conditions which ensure that the initial value problems of (1.1) shall have a unique solution for every initial condition.

Received February 18, 1992; revised September 10, 1992.

AMS Subject Classification: $34 \mathrm{C} 05$

* This work was supported by National Natural Science Foundation of China

Key words: nonlinear differential system; periodic solution; existence. 
The system (1.1) is a generalization of the following Liénard system

$$
\begin{aligned}
& d x / d t=y \\
& d y / d t=-y f(x)-g(x)
\end{aligned}
$$

For the system (1.2), the question of the existence of periodic solutions has been widely investigated by many authors, and many good results have been obtained (see e.g. [1] [2] for an extensive bibliography and also [3]-[5]). It is worthwhile to generalize these results to more general nonlinear system. We shall be concerned here with sufficient conditions for (1.1) to possess non-trivial periodic solutions.

In this paper we use some new techniques to deal with the existence of nontrivial periodic solutions of (1.1) and obtain some new results (see Theorems 1-3 in Section 2). If applying these new results to the system (1.2), from Remarks in Section 2, one will find that our results generalize some known results in this direction, and, in certain important aspects our conditions are less restrictive. For example, in our results, $f(x)$ is allowed to change its sign infinitely many times, in particular, our theorem 1 and theorem 3 give conditions under which the system (1.1) has at least a non-trivial periodic solution without the traditional assumptions that $G( \pm \infty)=+\infty$ and $f(x)$ is positive (or nonnegative) when $|x|$ is large. As is well known these assumptions play important roles in the proof of many known results on the existence of periodic solutions of (1.2). In Section 2, we also give several simple examples to illustrate cases in which the new criteria can be applied whereas criteria obtained in [1]-[7] fail.

For ease of exposition, throughtout the paper, we will use the following notations

$$
F(x)=\int_{0}^{x} f(s) d s \quad G(x)=\int_{0}^{x} g(s) d s
$$

and we make here the following assumptions

(H1) $y e(y)>0$ for all $y \neq 0 ; x g(x)>0$ for all $x \neq 0$.

(H2) For $0<|x| \ll 1, f(x) \leq 0$ and $\not \equiv 0$. 


\section{Resulits and Remarks}

Theorem 1. Assume (H1) and (H2) are satisfied. Moreover, assume the following conditions (H3)-(H6) also hold

(H3) $\varliminf_{x \rightarrow+\infty} F(x)>-\infty, \varlimsup_{x \rightarrow-\infty} F(x)<+\infty$. (where $\varlimsup$ im and $\underline{\text { lim represent }}$ limsup and liminf respectively.)

(H4) $\lim _{x \rightarrow+\infty}(F(x)+G(x))=+\infty ; \lim _{x \rightarrow-\infty}(-F(x)+G(x))=+\infty$.

(H5) $\varliminf_{g \rightarrow \pm \infty}|e(y)|>0$.

(H6) $\stackrel{g \rightarrow \pm}{\text { There exist } a \geq 0 \text { and }} q(x) \in C^{1}\left((-\infty,-a], R^{+}\right)$(or $q(x) \in C^{1}([a$, $\left.+\infty), \mathbb{R}^{-}\right)$) such that

$$
e(q(x))\left[f(x)+q^{\prime}(x)\right]+g(x) \geq 0 \quad(\leq 0)
$$

for all $x \leq-a(x \geq a)$.

Then there is at least one non-trivial periodic solution for the system (1.1).

Theorem 2. Let (H1) and (H2) hold, and the following conditions (H7)(II9) be satisfied

$(\mathrm{H} 7) e(y)$ is monotone increasing, and $e( \pm \infty)= \pm \infty$.

(H8) There exist $a>0$ and $b>0$ such that $f(x) \geq 0$ for all $x \notin(-a, b)$.

(H9) $F(b)>F(-a) ; G( \pm \infty)=+\infty$.

Then there exists at least one non-trivial periodic solution of the system (1.1).

Theorem 3. If the conditions ( $\mathrm{H} 1)-(\mathrm{H} 4)$ and $(\mathrm{H} 7)$ are satisfied, and the following condition ( $\mathrm{H} 10)$ holds

(H10) There exists $a>0$ such that $F(a)>F(-a)$, and for all $x \geq a$, $g(-x)=-g(x)$ and $f(x)+f(-x) \geq 0$.

Then the system (1.1) has at least one non-trivial periodic solution.

Remark 1. Theorems 1-3 are still valid if we consider the system

$$
\begin{aligned}
& d x / d t=e(y-F(x)) \\
& d y / d t=-g(x)
\end{aligned}
$$


which is equivalent to the system (1.1). In fact the nonlinear transformation

$$
T(x, y)=(x, y+F(x))
$$

carries system (2.1) into system (1.1) preserving the $x$-coordinates.

Remark 2. (H2) is only used to guarantee that the stationary point $(0,0)$ is local repulsive, therefore, the assumption (H2) may be replaced by; any set of assumptions which guarantee that the origin is repulsive. By the way, using theorem 1-3 and the change of coordinates $x^{*}=x, y^{*}=-y$, and $t^{*}=-t$, one may consider the case "For $0<|x| \ll 1, f(x) \geq 0$ and $\not \equiv 0$." which implies the origin is a local sink.

Remark 3. Obviously, if $F( \pm \infty)= \pm \infty$, then (H3) holds naturally, and (H4) also holds when $x g(x)>0$ for all $x \neq 0$.

If $G( \pm \infty)=+\infty$, then (H4) holds when (H3) is satisfied.

It is easy to see that (H8) implies (H3).

If the condition (e) of Theorem in [3] "there exist $h \geq \delta$ and $b>0$ such that $f(x)+|g(x)|>b>0$ if $|x|>h$." is satisfied, then (H4) holds when the condition " $x g(x)>0$ for all $x \neq 0$ " holds.

Remark 4. If $\varliminf_{x \rightarrow-\infty} f(x)>0$ (or $\varliminf_{x \rightarrow+\infty} f(x)>0$ ) and $\varlimsup_{x \rightarrow-\infty}\{|g(x)|$ $\left./ f(x)\}<\sup _{y \in R^{+}}\{e(y)\} \stackrel{x \rightarrow-\infty}{\text { (resp. }} \varlimsup_{x \rightarrow+\infty}\left\{|g(x)| / f^{\prime}(x)\right\}^{\infty}<\sup _{y \in R^{-}}\{-e(y)\}\right)$, then (H6) is satisfied if $x g(x)>0$ for all $x \neq 0$. In fact, the above hypothesis implies that there exist $a>0$ and $K>0$ such that $f(x)>0$ and $e(K) \geq|g(x)| / f(x)$ (resp. $-e(-K) \geq|g(x)| / f(x))$ for all $x \leq-a$ (resp. $x \geq a)$. Hence, let $q(x)=K$ (resp. $=-K)$, then (H6) follows.

Remark 5. For the system (1.2), (H5) holds naturally. Therefore, Theorem 1 of [5] is a special case of our Theorem 2 for $e(y)=y$, and, by the above Remarks, one can find that our Theorem 1 generalize the result of [3] and relax some conditions imposed in [3].

In order to compare our results with those obtained in [1]-[7], let us now see several simple examples 
Example 1. The system

$$
\begin{aligned}
& d x / d t=y^{2 n+1} \\
& d y / d t=-y^{2 n+1}\left(x^{3}+2 x^{2}-x-2\right) e^{x}-x^{5}\left(e^{x}+e^{-x}\right)
\end{aligned}
$$

has at least a non-trivial periodic solution for any nonnegative integer $n$.

Proof. For (2.2), we have $e(y)=y^{2 n+1}, f(x)=\left(x^{3}+2 x^{2}-x-2\right) e^{x}$ and $g(x)=x^{5}\left(e^{x}+e^{-x}\right)$. Obviously, (H1), (H2) and (H7) are satisfied, and $g(-x)=-g(x)$ for every $x \in R$.

By some simple calculuses, we have

$$
\begin{aligned}
& F(x)=\left(x^{3}-x^{2}+x-3\right) e^{x}+3 \\
& G(x)=\left(x^{5}+20 x^{3}+120 x\right)\left(e^{x}-e^{-x}\right)+\left(-5 x^{4}-60 x^{2}-120\right)\left(e^{x}+e^{-x}\right)+240
\end{aligned}
$$

Thus, it is easy to see that $F(+\infty)=+\infty$ and $F(-\infty)=3$ and $G( \pm \infty)=+\infty$. Therefore, (H3) and (H4) hold.

Again since

$$
\begin{aligned}
F(x)-F(-x) & =\left(x^{3}+x\right)\left(e^{x}+e^{-x}\right)+\left(e^{-x}-e^{x}\right)\left(x^{2}+3\right) \\
f(x)+f(-x) & =\left(x^{2}-1\right)(x+2) e^{x}+\left(x^{2}-1\right)(2-x) e^{-x}
\end{aligned}
$$

consequently

$$
\lim _{x \rightarrow+\infty}(F(x)-F(-x))=+\infty \text { and } \lim _{x \rightarrow+\infty}(f(x)+f(-x))=+\infty
$$

this implies that there exists $a>0$ such that $F(a)>F(-a)$ and $f(x)+f(-x)>0$ for all $x \geq a$, and hence (H10) holds. It therefore follows from Theorem 3 that the assertion of Example 1 is true.

Remark 6. For the system (2.2), it should be noted that $f(x)<0$ for $x<-2$ and $f(x)>0$ for $x>1$, therefore, the result of [3] cannot be invoked to produce a periodic solution of (2.2) for the special case $n=0$. Again since $G( \pm \infty)=+\infty$ and $F(-\infty)=3$, consequently, Theorem 3 and Corollaries 1 and 2 of [4] are also invalid for $n=0$. 
Notice also that the proof of Example 1 remains valid if $y^{2 n+1}$ is replaced by any function $e(y)$ which satisfies (H1) and (H7).

Example 2. The following systems

$$
\begin{aligned}
& d x / d t=\operatorname{arctg} y \\
& d y / d t=-(\operatorname{arctg} y)\left(x^{2} \sin ^{2} x-2 x-2\right)-2 x /\left(1+x^{2}\right)^{2}
\end{aligned}
$$

and

$$
\begin{aligned}
& d x / d t=y \\
& d y / d t=-y\left(x^{2} \sin ^{2} x-2 x-2\right)-2 x /\left(1+x^{2}\right)^{2}
\end{aligned}
$$

have non-trivial periodic solution.

Proof. For (2.3) and (2.4), it is easy, to show that (H1) and (H2) are satisfied, and

$$
F(x)=\frac{1}{6} x^{3}-\frac{1}{4} x^{2} \sin 2 x-\frac{1}{4} x \cos 2 x+\frac{1}{8} \sin 2 x-x^{2}-2 x
$$

This implies $F( \pm \infty)= \pm \infty$, from Remark 3 it follows that $(\mathbb{H} 3)$ and $(\mathbb{H} 4)$ hold.

On the other hand, for (2.3) and (2.4), we have respectively $\lim |e(y)|=$ $\lim _{y \rightarrow \infty}|\operatorname{arctg} y|=\pi / 2$ and $\underset{y \rightarrow \infty}{\lim }|e(y)|=\varliminf_{y \rightarrow \infty}|y|=+\infty$, consequently, (H5) also

Again since, for (2.3) and (2.4), we have $\varliminf_{x \rightarrow-\infty} f(x)=+\infty$, and $\varlimsup_{x \rightarrow-\infty}\{|g(x)|$ $\mid f(x)\}=0<\sup _{y \in R^{+}}\{e(y)\}$, therefore, it follows from Remark 4 that (H6) is satisfied. Thus, according to Theroem 1, the proof of Example 2 is completed.

Remark 7. For the system (2.4), it is easy to see that $f(x)$ is oscillatory on the interval $(0,+\infty)$, and, $\varlimsup_{x \rightarrow+\infty} f(x)=+\infty, \underset{x \rightarrow+\infty}{\varliminf_{i m}} f(x)=-\infty$, so the result of Villari [3] will fail. Again since $g(x)=2 x /\left(1+x^{2}\right)^{2} \rightarrow 0$ (as $x \rightarrow \infty$ ) and $G(x)=x^{2} /\left(1+x^{2}\right) \rightarrow 1($ as $x \rightarrow \infty)$, therefore, Theorems of A. B. Dragilev and A. F. Filippov (see [1] Theorems 5.1 and 5.3), Theorem 3 of Villari [5] and the results of Huang [6] and Ding [7] cannot be applied. 
We also would like to point out that, according to Remark $4, y$ on the right-hand side of (2.4) can be replaced by any function $e(y)$ satisfying (H5) and (H1).

\section{Proofs of Theorems}

We first prove a lemma.

Lemma. 1. If $(\mathrm{H} 1)$ and ( $\mathrm{H} 2)$ are satisfied, then origin is a unique singular point of (1.1), and is local repulsive.

Proof. According to ( $\mathrm{H} 1)$, it is easy to see that the origin is the only singular point of (1.1), and for $0<C_{0} \ll 1$, the curve

$$
V(x, y)=G(x)+\int_{0}^{y} e(s) d s=C_{0}
$$

is a closed curve surrounding the origin. Thus, it is obvious, based on $d V /\left.d t\right|_{(1.1)}$ $=-e^{2}(y) f(x)$ and $(\mathrm{H} 2)$, that the origin is local repulsive, and the lemma is proved.

By Lemma 1, if one can prove that there exists a bounded positive semitrajectory for (1.1) under the conditions of Theorem 1 or 2 or 3 , then the Poincaré-Bendixson theorem can be invoked to produce a non-trivial soltion of (1.1).

(I). Proof of Theorem 1

We first consider the case $q(x) \in C^{1}\left((-\infty,-a], R^{+}\right)$in (H6).

Let $x_{0}<-a, y_{0}>q\left(x_{0}\right)$, and $T_{A}^{+}$be a positive semi-trajectory of (1.1) passing through point $A\left(x_{0}, y_{0}\right)$ at time $t_{0}$, and let its parametric equation be

$$
x=x(t), \quad y=y(t) \quad t \geq t_{0}
$$

Since $y e(y)>0$ for all $y \neq 0$, it follows, when $t$ increases, that the point $(x(t), y(t))$ moves along $T_{A}^{+}$from the left (resp. right) to the right (resp. left) 
in the $y>0$ (resp. $y<0$ ) half-plane. Now we prove that $T_{A}^{+}$must intersect the positive $x$-axis.

Suppose that $T_{A}^{+}$doesn't intersect the positive $x$-axis for all $t \geq t_{0}$, then we have $y(t)>0$ for every $t \geq t_{0}$, and when $t \rightarrow+\infty, T_{A}^{+}$must belong to one of the following three possible cases

(i). $\varlimsup_{t \rightarrow+\infty} x(t)<+\infty, \varlimsup_{t \rightarrow+\infty} y(t)=+\infty$.

(ii). $\varlimsup_{t \rightarrow+\infty} x(t)=+\infty, \varlimsup_{t \rightarrow+\infty} y(t)=+\infty$.

(iii). $\varlimsup_{t \rightarrow+\infty} x(t)=+\infty, \varlimsup_{t \rightarrow+\infty} y(t)<+\infty$.

Integrating $d x / d t=e(y)$ along $T_{A}^{+}$from $t_{0}$ to $t$, we obtain

$$
x(t)=x\left(t_{0}\right)+\int_{t_{0}}^{t} e(y(t)) d t
$$

Thus, it follows from (H5) that $\varlimsup_{t \rightarrow+\infty} x(t)=+\infty$ when $\varlimsup_{t \rightarrow+\infty} y(t)=+\infty$, this means that the case (i) is impossible.

If the case (ii) or (iii) holds, then there exists $t_{1}>t_{0}$ such that $x\left(t_{1}\right)=0$ and $x(t)>0$ for all $t>t_{1}$. Thus, $g(x) / e(y) \geq 0$ for all $t \geq t_{1}$, it follows that

$$
\begin{aligned}
y(t) & =y\left(t_{1}\right)-\int_{0}^{x} f(s) d s-\int_{0}^{x} \frac{g(s)}{e(y(s))} d s \\
& \leq y\left(t_{1}\right)-F(x(t)) \quad\left(\text { as } \quad t \geq t_{1}\right)
\end{aligned}
$$

Using (H3) and the above inequality, it is not difficult to show $\varlimsup_{t \rightarrow+\infty} y(t)<+\infty$, consequently, the case (ii) is also impossible.

Assume the case (iii) holds, then there exists $P>0$ such that $0<y(t) \leq P$ for all $t \geq t_{0}$.

Let $Q>\max \left\{\begin{array}{l}\max \\ 0 \leq y \leq P\end{array} e(y), \quad 1\right\}$, then, for all $t \geq t_{1}$, we have

$$
\begin{aligned}
y(t) & =y\left(t_{1}\right)-\int_{0}^{x} f(s) d s-\int_{0}^{x} \frac{g(s)}{e(y(s))} d s \\
& \leq y\left(t_{1}\right)-F(x)-\frac{1}{Q} G(x)
\end{aligned}
$$

Again according to (II), there exists $k>0$ such that $F(x)>-k$ for all $x \geq 0$. On the other hand, (H4) implies that there exists $x^{*}>0$ such that $F(x)+G(x) \geq$ 
$Q y\left(t_{1}\right)+(Q-1) k$ for all $x \geq x^{*}$, i.e., $G(x) \geq Q y\left(t_{1}\right)+(Q-1) k-F(x)$ for all $x \geq x^{*}$. Substituting this into (3.1), we obtain

$$
y(t) \leq-\frac{Q-1}{Q}(F(x)+k)<0 \quad\left(\text { as } x \geq x^{*}\right)
$$

This contradicts the fact that $y(t)>0$ for all $t \geq t_{0}$, the contradition illustrates that the case (iii) is also impossible. Therefore, $T_{A}^{+}$must intersect the positive $x$-axis at some point $B$.

By using a exactly similar fashion, we can prove that when $t$ increases continously, $T_{A}^{+}$must also intersect the negative $x$-axis at some point $C\left(x_{C}, 0\right)$.

If $x_{C} \geq-a$, by (H1) and the uniqueness of solutions for the initial value problem associated with system (1.1), it is easy to see that $T_{A}^{+}$is bounded. (see Fig. 1).

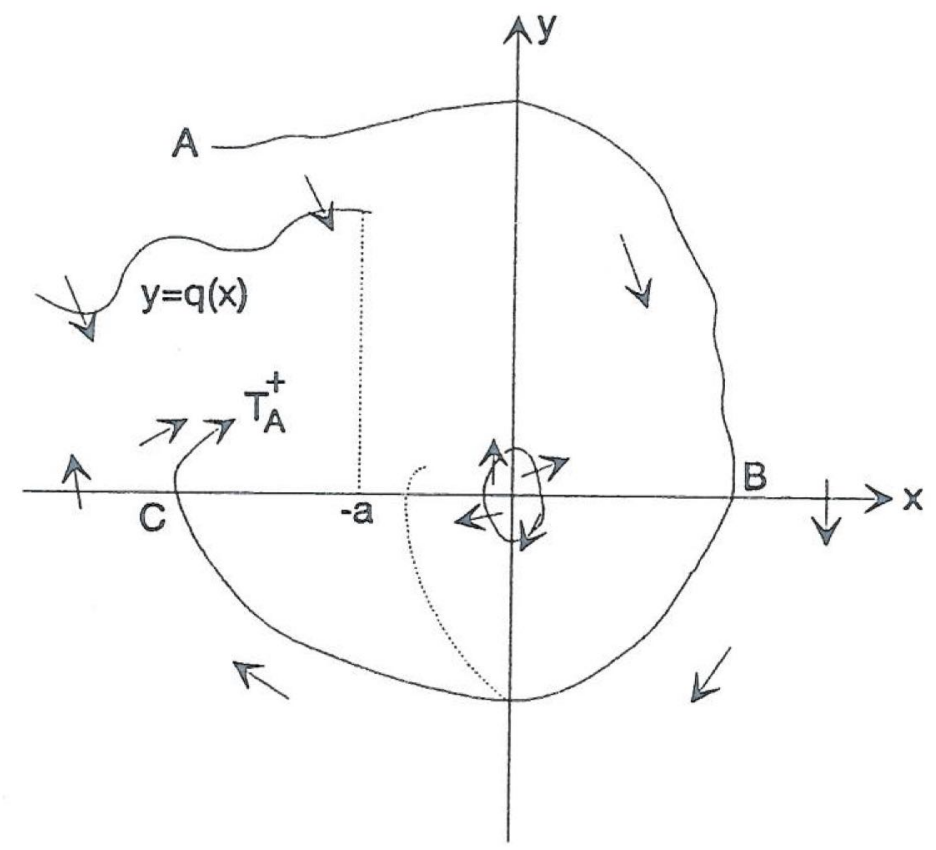

Figure 1

If $x_{C}<-a$, using (H6), on the curve $y=q(x)$, we have

$$
\frac{-e(y) f(x)-g(x)}{e(y)}-\frac{d q(x)}{d x} \leq 0 \quad(\text { as } x \leq-a)
$$

Consequently, $T_{A}^{+}$cannot cross the curve $y=q(x)$ as $x \leq-a$. Arguing as in the case $x_{C} \geq-a$ we can know that $T_{A}^{+}$is also bounded. 
For the case $q(x) \in C^{1}\left([a,+\infty), R^{-}\right)$in (H6), we choose $x_{0}>a y_{0}<q\left(x_{0}\right)$, by the same argument that was used to analyze $T_{A}^{+}$, we can prove that the positive semi-trajectory which starting at point $\left(x_{0}, y_{0}\right)$ must be bounded.

The Poincaré-Bendixson theorem now implies that system (1.1) has at least one non-trivial periodic solution.

(II). Proof of Theorem 2

Let

$$
\begin{aligned}
M & =\max \{|g(x)| \mid-a \leq x \leq b\} \\
N & =\max \{|f(x)| \mid-a \leq x \leq b\}
\end{aligned}
$$

Using (H7) and (H9), we may choose $W>0$ such that

$$
F(-a)-F(b)+\frac{2 M(b+a)}{\min \{e(W),|e(-W)|\}}<0
$$

Again let

$$
\begin{aligned}
& \Delta=W+\left[N+\frac{M}{\min \{e(W),|e(-W)|\}}\right](b+a) \\
& V(x, y)=G(x)+\int_{0}^{y} e(s) d s \\
& C_{*}>\max \{V(-a, \Delta), V(-a,-\Delta), V(b, \Delta), V(b,-\Delta)\}
\end{aligned}
$$

By (H1) and (H7) and (H9), it is clear that $V(x, y)=C_{*}$ is a closed curve surrounding the origin, and from our choice of $C_{*}$, it follows that $V(x, y)=C_{*}$ contains the region $\{(x, y)|-a \leq x \leq b| y \mid, \leq \Delta\}$ in its interior. Thus, the curve $V(x, y)=C_{*}$ must intersect the lines $x=-a$ and $x=b$ in the $y>0$ and $y<0$ half-plane. Let their intersection points be $A\left(-a, y_{A}\right), B\left(b, y_{B}\right)$, $C\left(b, y_{C}\right)$ and $D\left(-a, y_{D}\right)$ (see Fig.2), where $y_{A}, y_{B}>\triangle, y_{C}, y_{D}<-\triangle$. Again let $T_{A}^{+}: y=y(x)$ be a positive semi-trajectory of (1.1) starting at $A$ at time $t_{0}$. Using a proof similar to that used for Theorem 1 , we can prove that $T_{A}^{+}$cannot always stay in the region $\{(x, y) \mid-a \leq x<b, y \geq W\}$ for all $t \geq t_{0}$. Following this, we will show that $T_{A}^{+}$must intersect the line $x=b$ at a point $B *\left(b, y_{B^{*}}\right)$, and $y_{B^{*}} \geq W$. 


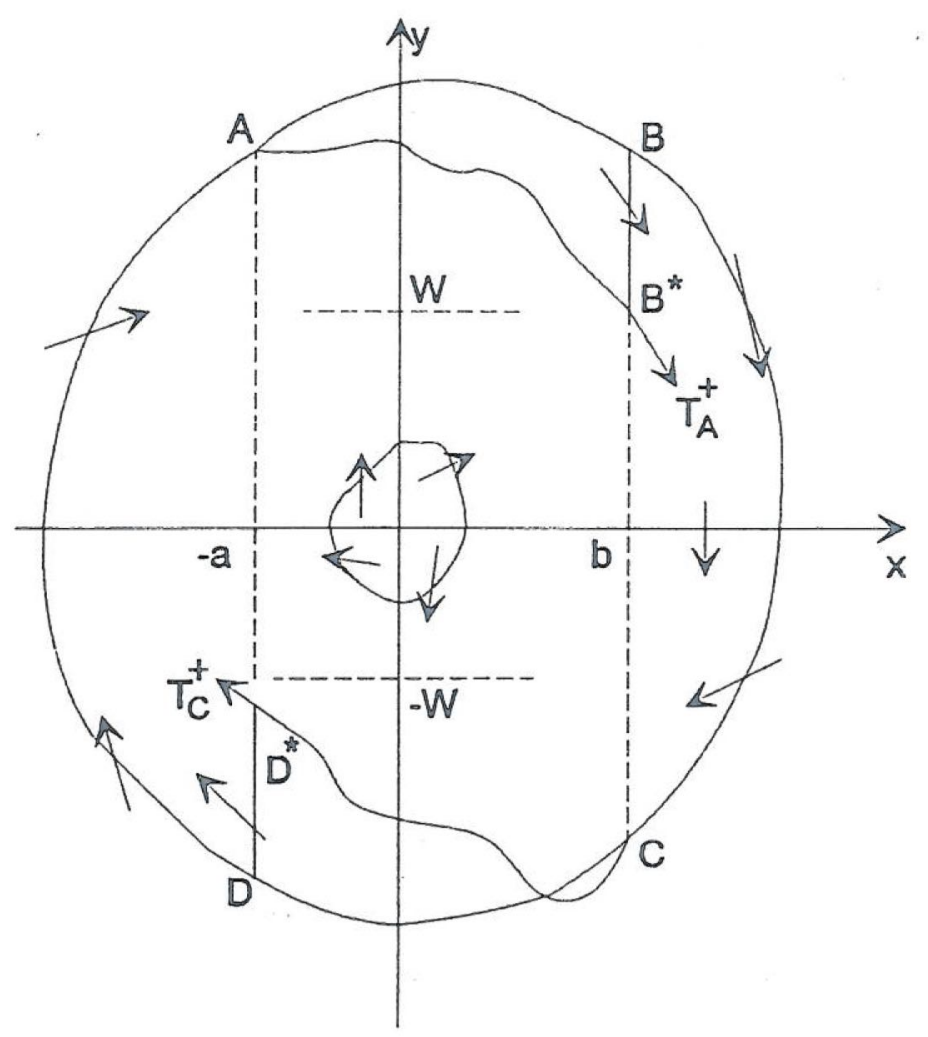

Figure 2

Otherwise, there exists $d \in(-a, b)$ such that $y(d)=W$ and $y(x)>W$ for all $x \in(-a, d)$. Again since

$$
\frac{d y}{d x}=-f(x)-\frac{g(x)}{e(y)}
$$

Integrating (3.2) along $T_{A}^{+}$on $[-a, d]$, we obtain

$$
\begin{aligned}
W-y_{A} & =-\int_{-a}^{d} f(x) d x-\int_{-a}^{d} \frac{g(x)}{e(y(x))} d x \\
& \geq-N(d+a)-\frac{M}{e(W)}(d+a) \geq-\left[N+\frac{M}{e(W)}\right](b+a)
\end{aligned}
$$

Therefore

$$
y_{A} \leq W+\left[N+\frac{M}{e(W)}\right](b+a) \leq \triangle
$$

This contradicts $y_{A}>\triangle$. Hence $T_{A}^{+}$must intersect the line $x=b$ at some point $B *\left(b, y_{B^{*}}\right)$ and $y_{B^{*}} \geq W$. 
Next we further prove $y_{B^{*}}<y_{B}$. Notice that $V(x, y)=C_{*}$ is a integral curve of the system

$$
d x / d t=e(y) \quad d y / d t=-g(x)
$$

so along this curve we have

$$
y_{B}-y_{A}=-\int_{-a}^{b} \frac{g(x)}{e(y(x))} d x \geq \frac{M(b+a)}{-e(W)}
$$

On the other hand, integrating (3.2) along $T_{A}^{+}$from $A$ to $B^{*}$, we obtain

$$
y_{B^{*}}-y_{A} \leq F(-a)-F(b)+\frac{M(b+a)}{e(W)}<-\frac{M(b+a)}{e(W)}
$$

From (3.3) and (3.4) it follows easily $y_{B^{*}}<y_{B}$.

By an analogous argument, one can prove that the positive semi-trajectory $T_{C}^{+}$starting at $C$ must intersect the line $x=-a$ at a poirt $D^{*}\left(-a, y_{D^{*}}\right)$ in the $y<0$ half-plane, and $y_{D}<y_{D^{*}} \leq-W$.

From (H8) it follows, along the curve $V(x, y)=C_{\text {* }}$, that $d V /\left.d t\right|_{(1.1)}=$ $-e^{2}(y) f(x) \leq 0$ for all $x \notin(-a, b)$. Again since $y e(y)>0$ for every $y \neq 0$, consequently, it is easy to see that $T_{A}^{+}$and $T_{C}^{+}$cannot cross the closed curve $\overparen{A B^{*}} \cup \overline{B^{*} B} \cup \overparen{B C} \cup \overparen{C D^{*}} \cup \overline{D^{*} D} \cup \overparen{D A}$ (see Fig. 2) into its exterior. This implies that $T_{A}^{+}$and $T_{C}^{+}$are bounded, and the proof is completed.

(III). Proof of Theorm 3

Let

$$
M^{*}=\max \{|g(x)||| x \mid \leq a\} \quad N^{*}=\max \{|f(x)||| x \mid \leq a\}
$$

According to (H7) and (H10), we may choose $W^{*}>0$ such that

$$
F(-a)-F(a)+\frac{2 a M^{*}}{\min \left\{e\left(W^{*}\right),\left|e\left(-W^{*}\right)\right|\right\}}<0
$$

Again let

$$
\Delta^{*}=W^{*}+2 a\left[N^{*}+\frac{M^{*}}{\min \left\{e\left(W^{*}\right),\left|e\left(-W^{*}\right)\right|\right\}}\right]
$$

In order to prove Theorem 3 , we first show two lemmas. 
Lemma 2. Let $y_{0}>\triangle^{*}, T^{+}: x=x(t), y=y(t)$ be a any positive semitrajectory starting at the point $\left(-a, y_{0}\right)\left(\right.$ or $\left.\left(a,-y_{0}\right)\right)$ at time $t_{0}$. Then, under the conditions of Theorem 3 , there exists $t_{1}>t_{0}$ such that $T^{+}$intersects the line $x=a($ resp. $x=-a)$ at time $t_{1}$, and $y(t)>W^{*}\left(\right.$ resp. $\left.y(t)<-W^{*}\right)$ for all $t \in\left[t_{0}, t_{1}\right)$, further, we also have $y\left(t_{1}\right)<y\left(t_{0}\right)=y_{0}$ (resp. $\left.y\left(t_{1}\right)>y\left(t_{0}\right)=-y_{0}\right)$.

Proof. We only consider the case that $T^{+}$starts at the point $\left(-a, y_{0}\right)$, since, in exactly the same way we can treat the case that $T^{+}$starts at the point $\left(a,-y_{0}\right)$.

By a similar argument that was used in (II) to prove that $T_{A}^{+}$intersects the line $x=b$ and $y_{B^{*}} \geq W$, we can prove that the two first assertions of Lemma 2 are true. Now we only prove $y\left(t_{1}\right)<y\left(t_{0}\right)=y_{0}$.

Integrating (3.2) along $T^{+}$from $-a$ to $a$, we obtain

$$
\begin{aligned}
y\left(t_{1}\right)-y_{0} & =-\int_{-a}^{a} f(x) d x-\int_{-a}^{a} \frac{g(x)}{e(y(x))} d x \\
& \leq-(F(a)-F(-a))+\frac{2 a M^{*}}{e\left(W^{*}\right)}<0
\end{aligned}
$$

This implies $y\left(t_{1}\right)<y\left(t_{0}\right)=y_{0}$.

Lemma 3. Let $h>a$, the trajectory $y=y(x)$ of (1.1) intersect both the lines $x=h$ and $x=-h$, and let $y(x)>0($ or $<0)$ for $x \in[-h, h]$. Then under the conditions of Theorem 3 , when $y(-a)>y(a)$, we have $y(-h)>y(h)$, and the function $z(h)=y(h)-y(-h)$ is monotone nonincreasing on $h \in(a,+\infty)$.

Proof. We only prove the case where $y(x)>0$ for all $x \in[-h, h]$, for the case $y(x)<0$, it can be treated in the same way.

Integrating (3.2) along the trajectory $y=y(x)$ on $[-h, h]$, we have

$$
z(h)=y(h)-y(-h)=-\int_{-h}^{h} f(x) d x-\int_{-h}^{h} \frac{g(x)}{e(y(x))} d x
$$

A simple calculation yields

$$
\begin{aligned}
\frac{d}{d h} z(h) & =-(f(h)+f(-h))-\left[\frac{g(h)}{e(y(h))}+\frac{g(-h)}{e(y(-h))}\right] \\
& \leq \frac{g(h)[e(y(h))-e(y(-h))]}{e(y(h)) e(y(-h))}
\end{aligned}
$$


Now let

$$
S(h)= \begin{cases}\frac{g(h)[e(y(h))-e(y(-h))]}{z(h) e(y(h)) e(y(-h))} & \text { when } z(h) \neq 0 \\ 0 & \text { when } z(h)=0\end{cases}
$$

Then the above inequality means that

$$
z^{\prime}(h) \leq S(h) z(h)
$$

where ' $=d / d h$.

Using (H1) and (H7), it is not diffcult to show $S(h) \geq 0$ for $h>a$, and hence $z^{\prime}(h) \leq 0$ as $z(h)=0$. Again since $S(h)$ is continuous at $h$ of $z(h) \neq 0$, thus, it follows from (3.5) that

$$
\frac{d}{d h}\left\{z(h) \exp \left[-\int_{a}^{h} S(\tau) d \tau\right]\right\} \leq 0
$$

Therefore

$$
z(h) \leq z(a) \exp \left[\int_{a}^{h} S(\tau) d \tau\right]=(y(a)-y(-a)) \exp \left[\int_{s}^{h} S(\tau) d \tau\right]<0
$$

Again using (3.5), we obtain $z^{\prime}(h) \leq 0$.

Thus, by what we have just proved, for any $h$ satisfying the conditions of Lemma 3 we have $z^{\prime}(h) \leq 0$. Hence $z(h)$ is monotone nonincreasing on $h$, it follows that $z(h)=y(h)-y(-h) \leq z(a)<0$ for $h>a$, i.e. $y(h)<y(-h)$, and the lemma is proved.

Now let us complete the proof of Theorem 3.

Let $A_{1}\left(-a, y_{A_{1}}\right)$ be a point on the straight line $x=-a$, with $y_{A_{1}}>\triangle^{*}$, and $T_{A_{1}}^{+}$be a positive semi-trajectory of (1.1) passing through $A_{1}$. From Lemma 2 it follows that $T_{A_{1}}^{+}$must intersect the line $x=a$ at some point $A_{2}$ in the half-plane $y>0$. Again using a proof similar to that used for Theorem 1 , we can prove that $T_{A_{1}}^{+}$must be clockwise and intersect the positive $x$-axis at some point $A_{3}$, and also intersect the line $x=a$ at some point $A_{4}\left(a, y_{A_{4}}\right)$ in the half-plane $y<0$.

Choose point $B_{1}\left(a, y_{B_{1}}\right)$ on the line $x=a$ such that $y_{B_{1}}<-\max \left\{\triangle^{*},\left|y_{A_{1}}\right|\right\}$. Let $T_{B_{1}}^{+}$and $T_{B_{1}}^{-}$be respectively the positive semi-trajectory and the negative 
semi-trajectory of (1.1) passing through $B_{1}$. By Lemma 2 we know that $T_{B_{1}}^{+}$must intersect the negative $y$-axis at a point $B_{2}$, also intersect the line $x=-a$ in the half-plane $y<0$. Let this intersection point be $B_{3}\left(-a, y_{B_{3}}\right)$, then $y_{B_{3}}>y_{B_{1}}$. Again arguing as in (I) we may prove that $T_{B_{1}}^{+}$must be clockwise and intersect the negative $x$-axis at a point $B_{4}$, and also intersect the line $x=-a$ again in the half-plane $y>0$. Let this intersection point be $B_{5}\left(-a, y_{B_{5}}\right)$.

If $y_{B_{5}} \leq y_{A_{1}}$, by the conditions, it is easy to see that $T_{B_{1}}^{+}$cannot cross the closed curve $\overparen{B_{1} B_{2} B_{3} B_{4} B_{5}} \cup \overline{B_{5} A_{1}} \cup \overparen{A_{1} A_{2} A_{3} A_{4}} \cup \overline{A_{4} B_{1}}$ (see Fig. 3 ) into its exterior. Thus, $T_{B_{1}}^{+}$must be bounded.

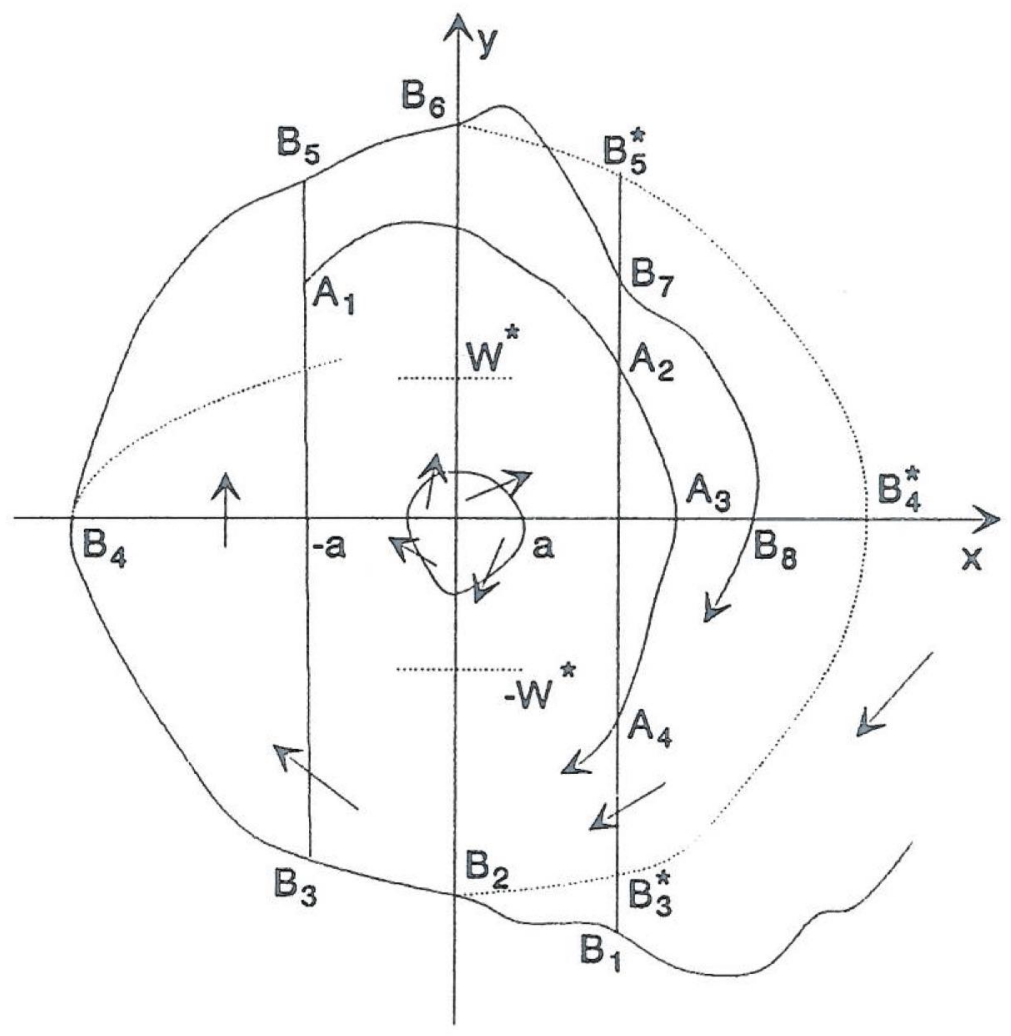

Figure 3

Now we assume $y_{B_{5}}>y_{A_{1}}$. By the same argument that was used to analyze $T_{A_{1}}^{+}$it follows that, when $t$ increases continously, $T_{B_{1}}^{+}$will intersect the positive $y$-axis for the first time at some point $B_{6}$, and intersect the line $x=a$ at some point $B_{7}\left(a, y_{B_{7}}\right)$ in the half-plane $y>0$, also intersect the positive $x$-axis at a point $B_{8}$. According to Lemma 2 we also have $W^{*} \leq y_{B_{7}}<y_{B_{5}}$. 
We now construct a symmetrical closed curve $\overparen{B_{2} B_{3} B_{4} B_{5} B_{6}} \cup \overparen{B_{6} B_{5}^{*} B_{4}^{*} B_{3}^{*} B_{2}}$ with respect to the $y$-axis (see Fig. 3), where $\overparen{B_{2} B_{3} B_{4} B_{5} B_{6}}$ is a segment of $T_{B_{1}}^{+}$. By Lemma 3 , it is easy to see that the segment $\overparen{B_{7} B_{8}}$ of $T_{B_{1}}^{+}$will be on the left-hand side of the segmental arc $\overparen{B_{5}^{*} B_{4}^{*}}$, and the part of $T_{B_{1}}^{-}$which belongs to the region $\{(x, y) \mid x>a, y<0\}$ must be on the right-hand side of the segmental $\operatorname{arc} \overparen{B_{3}^{*} B_{4}^{*}}$ (see Fig. 3). Therefore, by (H1), it is clear that $T_{B_{1}}^{+}$is bounded.

This completes the proof of Theorem 3.

\section{References}

[1] Ye Yanqian, "Theory of Limit Cycles (Second Edition)", Shanghai Science and Technology Press, 1984.

[2] Zang Zhifen, Ding Tongren, et al., "Qualitative Theory of Differential Equations", Press of Science, Peking, 1985.

[3] G. Villari, "Periodic Solutions of Liénard's Equation", J. Math. Anal. Appl., 86 (1982), 379-382.

[4] G. Villari, "On the Qualitative Behaviour of Solutions of Lienard Equation", J. Differetial Equations, 67 (1987), 269-277.

[5] G. Villari, "On the Existence of Periodic Solutions for Liénard's Equation", Nonlinear Anal., 7 (1983), 71-78.

[6] Huang Qichang, Shi Xufu, "On the Conditions for the Existence of Limit Cycles of Liénard's Equation", Science Bulletion, 27 (1982), 11, 645-646. [7] Ding Dazhong, "The Existence of Limit Cycles of Liénard's Equation", Chin. J. Appl.
Math., 7 (1984), 2, 166-174.

Department of Applied Mathematics, Hunan University, Changsha, Hunan 410082, China. 